\title{
Expression of canonical transient receptor potential channels in U-2 OS and MNNG-HOS osteosarcoma cell lines
}

\author{
FLORIAN LÄSSIG $^{1}$, ANJA KLANN ${ }^{2}$, SANDER BEKESCHUS $^{3}$, UWE LENDECKEL $^{1}$ and CARMEN WOLKE ${ }^{1}$ \\ ${ }^{1}$ Institute of Medical Biochemistry and Molecular Biology, University Medicine Greifswald, D-17475 Greifswald; \\ ${ }^{2}$ Institute of Forensic Medicine, University Medicine Greifswald; ${ }^{3}$ Zentrum für Innovationskompetenz (ZIK) Plasmatis, \\ Leibniz Institute for Plasma Science and Technology (INP), D-17489 Greifswald, Germany
}

Received September 10, 2020; Accepted January 4, 2021

DOI: $10.3892 / 01.2021 .12568$

\begin{abstract}
In U-2 OS and MNNG-HOS osteosarcoma cells, small interfering RNA-mediated knockdown of the angiotensin-(1-7) receptor, Mas, increases cell proliferation. Whether alterations in canonical transient receptor potential channels (TRPC) expression contribute to this effect is not clear. In the present study, a basic description of TRPC subtype expression in osteosarcoma cell lines was provided. The pharmacological modulators of the angiotensin-(1-7) receptor, Mas, AVE0991 (agonist), or D-Ala ${ }^{7}$-Ang-(1-7) (antagonist) were applied to elucidate a possible role of Mas in the regulation of TRPC mRNA levels. The contribution of other G-protein coupled receptors (GPCR) or receptor tyrosine kinases to TRCP expression was studied by applying the selective pharmacological blockers of either PI3 kinase or MEK/ Erk1/2 signaling, Ly294002 and PD98059. AVE0991 and D-Ala-Ang-(1-7) exhibited no or marginal effects on TRPC mRNA expression. Ly294002 provoked a 9.6- and 5.9-fold increase in the amounts of TRPC5 mRNA in MNNG-HOS and U-2 OS cells, respectively. Additionally, Ly294002 increased TRPC6 mRNA levels; however, it had no effect on TRPCs 1, 3 and 4. Administration of PD98059 increased the amounts of TRPC6 and TRPC4 2-fold. In conclusion, the present study demonstrated that Mas-dependent alterations in osteosarcoma cell line proliferation were not mediated by any changes in TRPC subtype gene expression. The data shows in principle, and consistent with the literature, that the signaling pathways examined can regulate the expression of TRPCs at the mRNA level. Therefore, direct and signaling pathway-specific phar-
\end{abstract}

Correspondence to: Dr Carmen Wolke, Institute of Medical Biochemistry and Molecular Biology, University Medicine Greifswald, Ferdinand-Sauerbruch-Strasse DZ7, D-17475 Greifswald, Germany

E-mail: carmen.wolke@med.uni-greifswald.de

Key words: osteosarcoma, renin-angiotensin-system, angiotensin-(1-7), Mas, canonical transient receptor potential channels, U-2 OS, MNNG-HOS macological targeting of TRPC subtypes may represent an option for improving the treatment of osteosarcoma.

\section{Introduction}

Canonical transient receptor potential (TRPC) channels are non-selective cation channels that are permeable to $\mathrm{Ca}^{2+}$ and, thus, under both physiological and pathological conditions facilitate $\mathrm{Ca}^{2+}$ entry into the cell. In humans, there are seven TRPCs known which can be divided into subgroups according to their degree of homology: C1/C4/C5, C3/C6/C7 and the inactive pseudogene $\mathrm{C} 2(1,2)$. TRPC channels exhibit baseline activity, which, however, can be increased via stimulation of G-protein coupled receptors (GPCR) or receptor tyrosine kinases. In this way, receptor agonist-dependent activation of Gq-PLC signal transduction pathways leading to IP3-mediated $\mathrm{Ca}^{2+}$ release from internal stores (SR; ER) represents the main mechanism of generating intracellular $\mathrm{Ca}^{2+}$ transients in nonexcitable cells via potentially any TRPC expressed. TRPCs are known to form heteromers in a large variety of cell types, which adds another level of complexity to the existing TRP network and complicates functional analyses. $\mathrm{Ca}^{2+}$ transients activate a variety of cellular effector processes, including proliferation, apoptosis, mitochondrial function, metabolism, migration, invasion and angiogenesis $(1,3,4)$. Furthermore, TRPC expression is altered in tumor tissue when compared to non-malignant tissue [reviewed in ref. 1]. Against this background, it is not surprising that TRPC channels have been linked to the development and progression of cancer and represent promising targets for the pharmacological treatment of different tumor entities (5-9). Although the importance of calcium for the proliferation of osteosarcoma cells is well established $(10,11)$, data on the expression and role of TRPC in osteosarcoma is scarce. It could be demonstrated in various cell models that TRPCs are involved in store operated calcium entry (SOCE) (12) and TRPC1 is one of the major components involved here (13). In line with the crucial role of $\mathrm{Ca}^{2+}$ for cytoskeletal reorganization, migration, differentiation, survival and apoptosis, and polarization, various TRPC members have been implicated in all these processes. Pharmacological inhibition or knock-down of TRPC1 was shown to reduce cell polarization of U-2 OS osteosarcoma cell line (14). It has been shown that the TRP family, including subfamilies TRPC, 
TRPM, and TRPV is highly expressed in human and murine osteosarcoma cell lines (15). Human osteosarcoma cell lines including U-2 OS were shown to share abundant expression of the TRPC members TRPC1, TRPC3 and TRPC6 (15).

We have shown previously that the alternative axis of the renin-angiotensin-system (RAS), ACE2/angiotensin-(1-7)/ Mas, is expressed and functional in U-2 OS and MNNG-HOS osteosarcoma cell lines. The knock-down of Mas substantially increased the proliferation of both cell lines (16). Whether calcium transients mediate these effects on osteosarcoma proliferation is not clear. Mas has been associated with $\mathrm{G}$ protein-mediated $\mathrm{Ca}^{2+}$-signaling in Mas-transfected HEK293 cells (17), but other signaling pathways such as MAPK and PI3K may well contribute to Mas-dependent alterations in osteosarcoma cell proliferation or TRPC expression. In this study, we provide a basic description of TRPC expression in both osteosarcoma cell lines and, in addition, address the question if the angiotensin-(1-7) receptor, Mas, is involved in the regulation of TRPC expression in osteosarcoma and if this has functional implications for cell migration. Furthermore, the contribution of other GPCRs or receptor tyrosine kinases to the regulation of TRCP expression is studied by applying the selective pharmacological blockers of either PI3 kinase or MEK/Erk1/2 signaling, Ly294002 and PD98059.

\section{Materials and methods}

Cell culture. The osteosarcoma cell lines U-2 OS and MNNG-HOS were purchased from the American Type Culture Collection (ATCC). U-2 OS cells were maintained in McCoy's 5A medium (PAA) and MNNG-HOS in MEM with Earle's Salt (Gibco; Thermo Fisher Scientific, Inc.), both supplemented with $10 \%$ fetal calf serum, $2 \mathrm{mM} \mathrm{L}$-glutamine, $1 \mathrm{mM}$ sodium pyruvate, $100 \mathrm{U} / \mathrm{ml}$ penicillin and $100 \mu \mathrm{g} / \mathrm{ml}$ streptomycin at $37^{\circ} \mathrm{C}$ in a $5 \% \mathrm{CO}_{2}$ humidified atmosphere. Cells were free from contamination with mycoplasma, as concluded from negative results obtained in regularly performed testing by means of the PCR Mycoplasma Testkit I/C (PromoCell).

Identification of cell lines was performed using the PowerPlex ${ }^{\circledR} 21$ System (Promega) and the software GeneMapper ${ }^{\circledR}$ ID-X v1.4 (Applied Biosystems; Thermo Fisher Scientific, Inc.), allowing automatic identification of the gender marker amelogenin and 20 highly polymorphic chromosomal markers (short tandem repeats) by fluorescent multiplex PCR. This marker panel was used to control an eventual sample mix-up or contamination during cell culture. Amelogenin and the eight STR markers incl. the specific alleles [CSF1PO, D13S317, D16S539, D5S818, D7S820, TH01, TPOX, vWA] are published for the cell lines U-2 OS (ATCC ${ }^{\circledR} \mathrm{HTB}^{\mathrm{N}}-96^{\mathrm{TM}}$ ) and MNNG/HOS Cl \#5 [R-1059-D] (ATCC ${ }^{\circledR}$ CRL-1547 ${ }^{\mathrm{TM}}$ ) in the American Type Culture Collection (ATCC) database (www. ATCC.org). The pattern of these eight markers observed in our cell lines corresponded to the published alleles.

$R N A$ preparation and reverse transcription-quantitative $P C R$ $(R T-q P C R)$. For experiments to investigate the influence of the Mas receptor agonist, AVE0991 $(1 \mu \mathrm{M}$; kindly provided by Sanofi-Aventis) or D-Ala-(Ang(1-7) (A779) $(1 \mu \mathrm{M}$, SigmaAldrich; Merck KGaA), on mRNA expression, the cells were seeded at a density of $5 \times 10^{5}$ cells/well of a 6 -well plate in a final volume of $3 \mathrm{ml}$ for $24 \mathrm{~h}$. The dosage is based on previous studies in which $1 \mu \mathrm{M}$ was identified to be the lowest effective dose with which insulin secretion from isolated islets can be maximally stimulated (18). RNA was prepared using the innuPrep RNA Mini Kit (Analytik Jena) according to the manufacturer's instructions. cDNA synthesis and RT-qPCR were performed in a CFX96 thermocycler (Bio-Rad Laboratories) as described recently (19). Quantitative analysis was performed using $\Delta \Delta \mathrm{Cq}$-method (20) included in the CFX Maestro RT-qPCR detection system software (Bio-Rad Laboratories). Size and purity of the PCR amplificates was determined by melt curve analysis. The amounts of mRNA were normalized to $\alpha$-tubulin mRNA. The relative expression levels of the individual TRPCs were compared on the basis of their Cq values using a calibration curve. Assuming 100\% PCR efficiency, 3.3 cycles lower $\mathrm{Cq}$ values correspond to a 10 -fold higher number of mRNA copies. Primers were designed with the aid of NCBI's Primer Designer and were obtained from Invitrogen (Thermo Fisher Scientific, Inc.). Primer-sequences [downstream (DS) and upstream (US)], size of amplified DNA fragments in base pairs (bp), and the optimized annealing temperatures are listed in Table I.

Cell migration assay. Cells were seeded at a density of $2 \times 10^{3}$ per well into 96-well plates (Eppendorf). After the cells were allowed to adhere, drugs (10 nM AVE0991, $1 \mu \mathrm{M}$ D-Ala ${ }^{7}$ (Ang(1-7), $3.5 \mu \mathrm{M}$ Pyr 3 (Bio-Techne $\mathrm{GmbH}$ ) and $35 \mu \mathrm{M}$ AC1903 (Bio-Techne $\mathrm{GmbH}$ ) or vehicle controls were added as indicated. Cell migration was analyzed using live-cell imaging and a high-content imaging device (Operetta CLS; PerkinElmer) every $20 \mathrm{~min}$ for at least $6 \mathrm{~h}$ at $37^{\circ} \mathrm{C}$ and $5 \% \mathrm{CO}_{2}$. The culture plates have a rim that can be filled with water to protect the cells from evaporation during live-cell imaging. Image acquisition was made using a 10x air (NA: 0.3) objective (Carl Zeiss). For each well, four fields of view were acquired. Image analysis was done by segmenting cells based on their digital phase-contrast signal intensity, followed by removing border objects and the calculation of kinetic properties based on signal overlaps between adjacent time points. For each well, approximately 500 cells were included in the analysis, which was done using Harmony 4.9 quantitative image analysis software algorithms (PerkinElmer).

Statistical analysis. Mann-Whitney tests were applied in the case of $n \geq 4$ when two groups were compared. When three or more groups were compared, ANOVA was used prior to the use of Tukey's post hoc test. Non-parametric data were illustrated as boxplots with medians, quartiles, and an interquartile range (IQR) $\pm 1.5 \times$ IQR with outliers as indicated (Tukey's method). P-values $<0.05$ were considered significant. All analyses were performed using GraphPad Prism version 6.03 (Graphpad Software, Inc.).

\section{Results}

TRPC family members and Mas are expressed in U-2 OS and MNNG-HOS cells. First, the expression of all human TRPC isoforms was analyzed at the mRNA level in both U-2 OS and MNNG-HOS cells under basic conditions by means of RT-qPCR. TRPCs 1, 3, 4, 5, 6 and 7 mRNAs could be detected 
Table I. Primers used for reverse transcription-quantitative PCR.

\begin{tabular}{|c|c|c|c|}
\hline Primer & Sequence $\left(5^{\prime}-3^{\prime}\right)$ & Amplicon size, bp & Annealing temperature, ${ }^{\circ} \mathrm{C}$ \\
\hline Mas-DS & CAATGCCGACTGGTACTTG & 407 & 62 \\
\hline Mas-US & ACATCTCACTGGCAGGAAC & & \\
\hline TRPC1-DS & CTGACAACCGTAGTCCAAAAG & 177 & 60 \\
\hline TRPC1-US & TTCTTGCTGGCGTGCGACAAG & & \\
\hline TRPC3-DS & GTCATTCTTGAACTCCTTCTC & 492 & 60 \\
\hline TRPC3-US & AAGAAGGAGAACCTGGCGCG & & \\
\hline TRPC4-DS & CTGCTTATCAAGGAGTATAGGA & 126 & 56 \\
\hline TRPC4-US & GTCTATGTTGGAGATGCTCTAT & & \\
\hline TRPC5-DS & GTTGTAACTTGTTCTTCCTGTC & 210 & 56 \\
\hline TRPC5-US & CAGTGAGGTAGAATTAGGTGAA & & \\
\hline TRPC6-DS & CCATCGTAACATTATAGACTCC & 191 & 55 \\
\hline TRPC6-US & GTTCAАТСТСТАСТССТАСТАС & & \\
\hline TRPC7-DS & GAACTTCCATTCCACATCTG & 194 & 64 \\
\hline TRPC7-US & GGTCCATATTCGGCTTATCT & & \\
\hline$\alpha$-tubulin-DS & CATTTCACCATCTGTTGGCTGGCTC & 528 & 58 \\
\hline$\alpha$-tubulin-US & CACCCGTCTTCAGGGTTCTTGGTTT & & \\
\hline
\end{tabular}

TRPC, canonical transient receptor potential channels; DS, downstream; US, upstream.

A

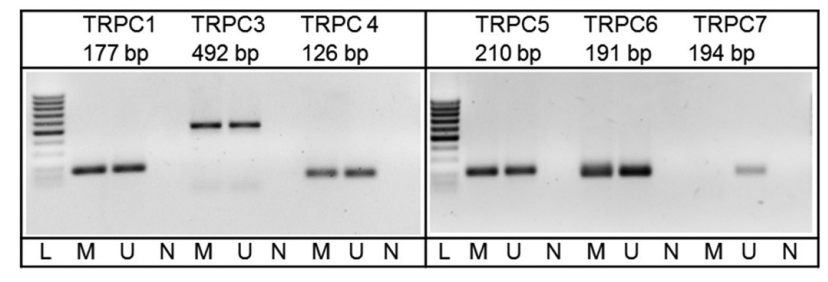

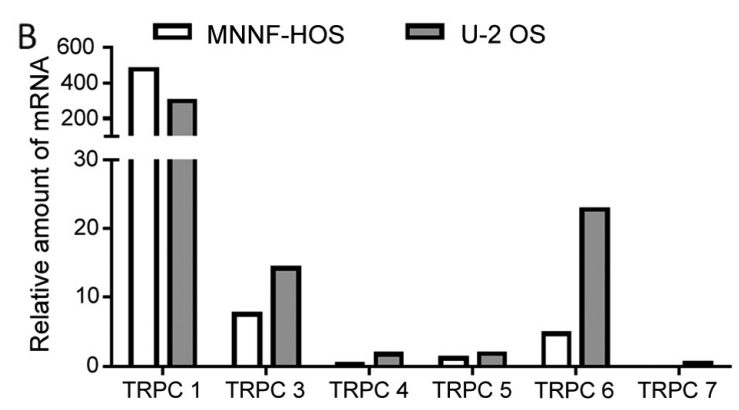

Figure 1. Expression levels of TRPC isoforms in MNNG-HOS and U-2 OS osteosarcoma cells. The expression levels of TRPC isoforms were verified at the mRNA level by RT-qPCR. (A) Formation of a unique amplicon of the correct size only was verified by agarose gel electrophoresis and melting curve analysis. mRNA for all TRPC isoforms investigated could be detected in U-2 OS cells. In MNNG-HOS cells, TRPC7 was not expressed. (B) Relative expression levels of TRPCs in osteosarcoma cell lines were determined by RT-qPCR of four pooled replicates each. RT-qPCR, reverse transcription-quantitative PCR; TRPC, canonical transient receptor potential channels. Bp, base pairs; L, 100 bp-ladder; M, MNNG-HOS; U, U-2 OS; N, negative control.

in comparable amounts in both cell lines. As illustrated in Fig. 1A, a single amplicon of the expected size was obtained for any TRPC isoform as confirmed by agarose gel electrophoresis as well as the melting curve obtained as part of the PCR protocol (data not shown). The level of expression of the individual TRPC isoforms is very different, but the 'TRPC profile' shows extensive similarity between the two cell lines (Fig. 1B). By far the highest mRNA levels could be detected for TRPC1 followed by TRPC6. TRPCs 3, 4 and 5 appeared to be moderately expressed (Fig. 1B). RT-qPCR analysis of TRPC7 provided Cq-values $>38$ in both cell lines and, thus, this isoform is regarded as practically not expressed in MNNG-HOS and U-2 OS cells.

Next, before studying the effects of the Mas agonist, AVE0991 or of the antagonist, D-Ala7-Ang-(1-7) (A779), on TRPC expression, mRNA expression of the Ang-(1-7) receptor, Mas, was confirmed by RT-qPCR. As previously observed (13) and shown in Fig. 2A, Mas mRNA could be detected in both U-2 OS and MNNG-HOS cells, with MNNG-HOS cells showing stronger expression levels than U-2 OS. In MNNG-HOS cells, the administration of AVE0991 led to a significant $[3.35(1.57 ; 7.16), \mathrm{p}<0.001]$ increase in Mas-mRNA levels (Fig. 2B). In U-2 OS cells, there was no such increase in Mas mRNA $\{1.4$ [1.29 (0.39; 3.89)], $\mathrm{p}=0.92\}$ (Fig. 2C). However, in response to the Mas receptor antagonist, D-Ala-Ang-(1-7), both MNNG-HOS and U-2 OS showed an induction of Mas mRNA expression [3.25 (0.83; 5.97), $\mathrm{p}<0.05$ or 2.41 (1.05; 5.42), $\mathrm{p}=0.12$ ] (Fig. 2B and C).

Effects of AVE0991 or D-Ala -Ang-(1-7) on the mRNA expression of TRPC family members. Next, the possible effects of a 24-h exposure of U-2 OS and MNNG-HOS cells to the Mas 
A

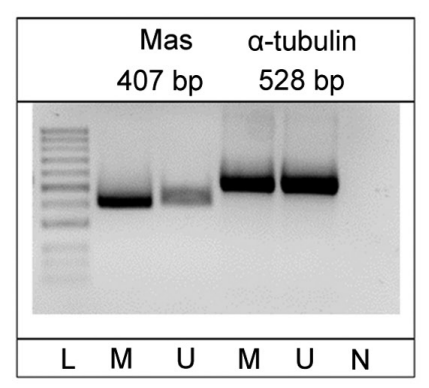

B

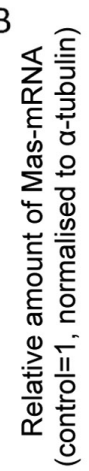

MNNG-HOS

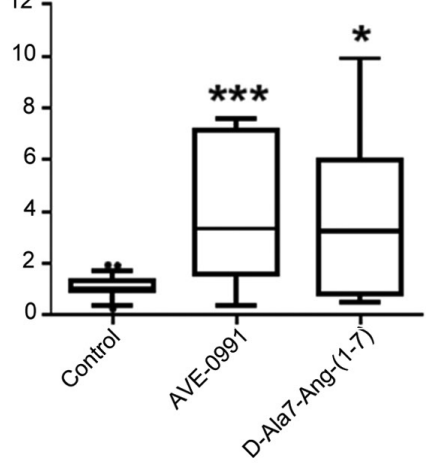

C

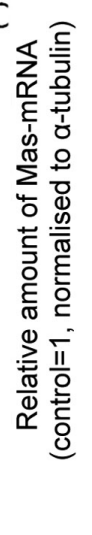

U-2 OS

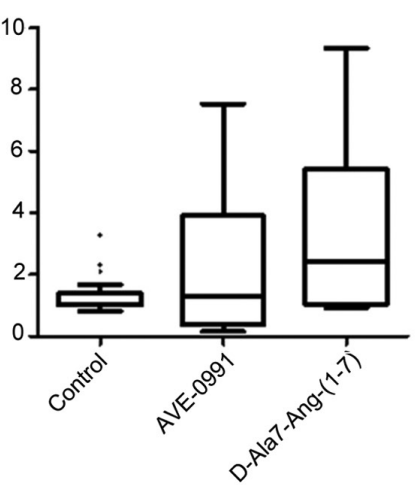

Figure 2. Expression levels of Mas in MNNG-HOS and U-2 OS osteosarcoma cells. (A) Reverse transcription-quantitative PCR analysis revealed the expression levels of Mas in MNNG-HOS and U-2 OS cell lines. The formation of a unique amplicon of the correct size only was verified by agarose gel electrophoresis and melting curve analysis. Mas-mRNA levels were subject to regulation in response to Mas receptor agonist, AVE0991, or antagonist, D-Ala -Ang-(1-7), in (B) MNNG-HOS osteosarcoma cells or (C) U-2 OS osteosarcoma cells ( $\mathrm{n}=8$; ${ }^{*} \mathrm{P}<0.05$, ${ }^{* * *} \mathrm{P}<0.001$ vs. control). bp, base pairs; L, 100 bp-ladder; M, MNNG-HOS; U, U-2 OS; N, negative control.
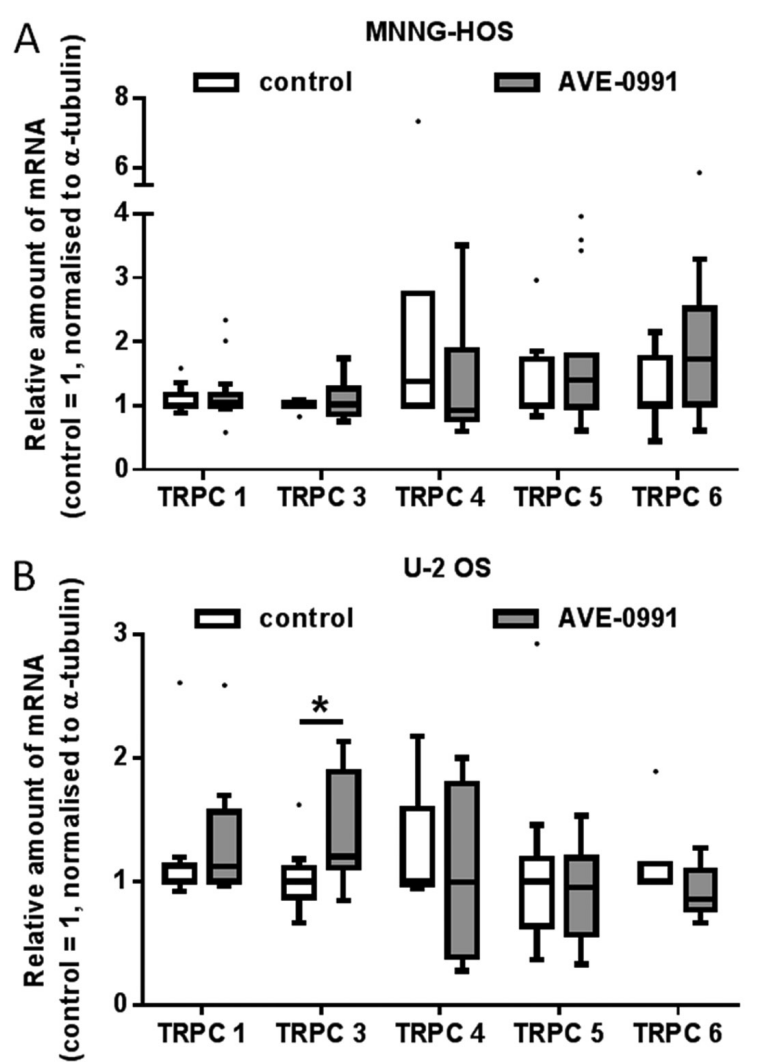

Figure 3. Effect of Mas receptor agonist, AVE0991, on the TRPC-mRNA content of (A) MNNG-HOS and (B) U-2 OS osteosarcoma cells. Cells were exposed to AVE0991 ( $1 \mu \mathrm{M})$ for $24 \mathrm{~h}$. Subsequently, mRNA content was analyzed by reverse transcription-quantitative PCR. Data are presented as boxplots with medians, quartiles and an IQR $\pm 1.5 \times$ IQR with outliers as indicated (Tukey's method; $\mathrm{n}=8$; " $\mathrm{P}<0.05$ ). IQR, interquartile range; TRPC, canonical transient receptor potential channels.

receptor agonist, AVE0991, or the antagonist D-Ala 7 -Ang-(1-7) on TRPC mRNA levels were investigated. In general, Mas receptor ligands provoked no or only minor alterations in TRPC mRNA expression, as summarized in Fig. 3 (AVE0991) and Fig. 4 (D-Ala -Ang-(1-7). In MNNG-HOS cells, AVE0991 led to a non-significant elevation of TRPC5 mRNA levels by
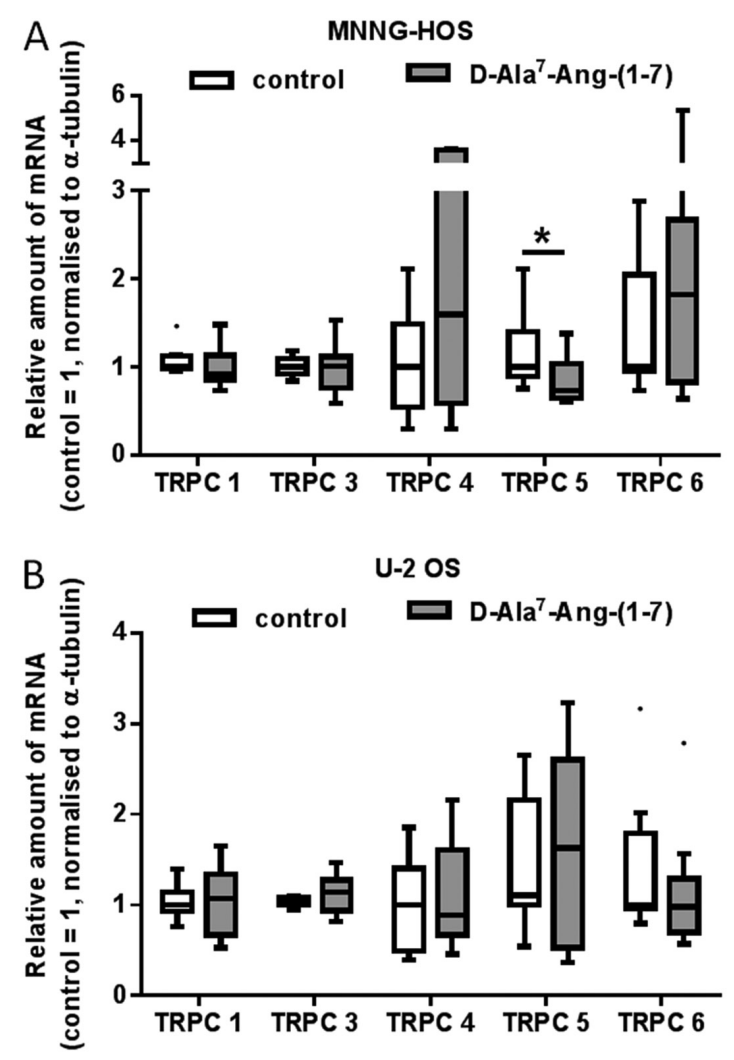

Figure 4. Effect of Mas receptor antagonist, D-Ala 7 -Ang-(1-7), on the TRPCmRNA content of (A) MNNG-HOS and (B) U-2 OS osteosarcoma cells. Cells were exposed to $1 \mu \mathrm{M}$ D-Ala ${ }^{7}$-Ang-(1-7) for $24 \mathrm{~h}$. Subsequently, mRNA content was analyzed by reverse transcription-quantitative PCR. Data are presented as boxplots with medians, quartiles and an IQR $\pm 1.5 \times \mathrm{IQR}$ with outliers as indicated (Tukey's method; $n=8 ;{ }^{*} \mathrm{P}<0.05$ ). IQR, interquartile range; TRPC, canonical transient receptor potential channels.

$70 \%$, whereas in U-2 OS cells, a small but significant increase in amounts of TRPC3 mRNA could be observed [1.2 (0.85; 2.13), $\mathrm{p}<0.05]$.

D-Ala ${ }^{7}$-Ang-(1-7) provoked a small but significant decrease of TRCP5 mRNA amounts in MNNG-HOS cells [0.73 (0.61; 1.38), $\mathrm{p}<0.05$ ]. 

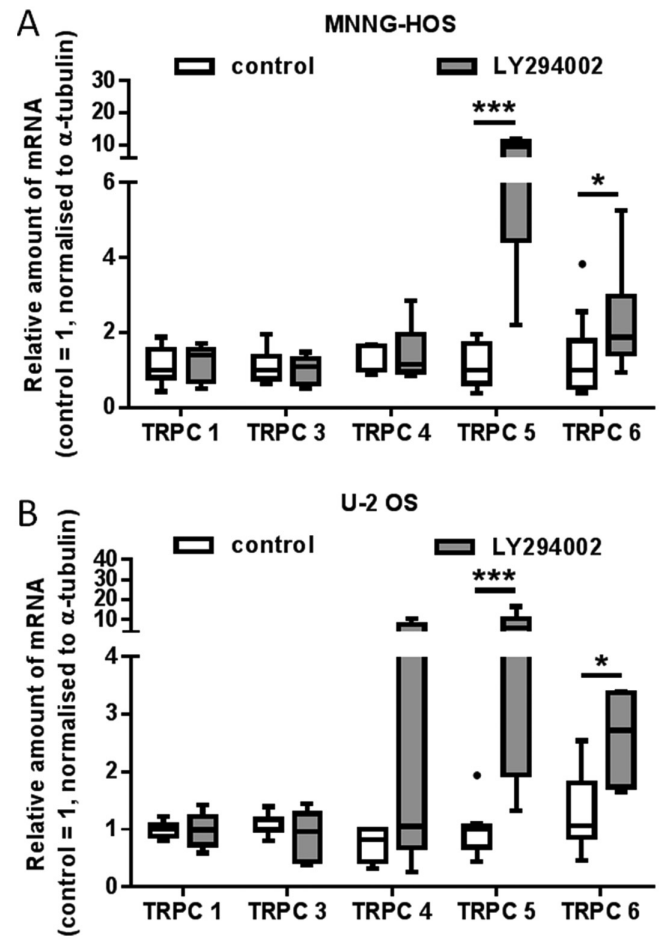

Figure 5. Effect of PI3 kinase inhibitor, LY294002, on the TRPC-mRNA content of (A) MNNG-HOS and (B) U-2 OS osteosarcoma cells. Cells were exposed to LY294002 (100 $\mu \mathrm{M})$ for $24 \mathrm{~h}$. Subsequently, mRNA content was analyzed by reverse transcription-quantitative PCR. Data are presented as boxplots with medians, quartiles and an IQR $\pm 1.5 \times$ IQR with outliers as indicated (Tukey method; $\mathrm{n}=8 ;{ }^{*} \mathrm{P}<0.05,{ }^{* * * *} \mathrm{P}<0.001$ ). IQR, interquartile range; TRPC, canonical transient receptor potential channels.

Effects of Ly294002 and PD98059 on the mRNA expression of TRPC family members. The exposure of MNNG-HOS and U-2 OS osteosarcoma cells to the selective pathway inhibitors Ly294002 or PD98059, respectively, led to substantial alterations in the mRNA expression levels of individual TRPC family members.

In detail, Ly294002 provoked a profound and highly significant increase in TRPC5 mRNA amounts in both MNNG-HOS [9.6 (2.192; 11.68), $\mathrm{p}<0.0001]$ and U-2 OS cells [5.87 $(1.32 ; 16.52) \mathrm{p}<0.001]$. Slightly weaker were the stimulatory effects of Ly294002 on the expression of TRPC6 in both cell lines; MNNG-HOS: 1.87[0.93; 5.27] p $<0.05$, U-2 OS: 2.72 $[1.64 ; 3.38], \mathrm{p}<0.05$. In contrast, the compound did not affect the mRNA levels of TRPC1, 3 and 4 (Fig. 5).

In MNNG-HOS cells, the administration of PD98059 led to an about 2.07-fold increase in mRNA levels of TRPC4 2.07 [1.13; 4.95], $\mathrm{p}<0.01$; and of TRPC6: 1.83 [1.04;4.90], $\mathrm{p}<0.05$ (Fig. 6A). An increase of TRPC4 and TRPC6 mRNA amounts in U-2 OS cells did not reach statistical significance (Fig. 6B). A small but significant increase in TRPC 3 mRNA amounts could be observed in U-2 OS cells: 1.29 [1.02; 2.44], $\mathrm{p}<0.05$, whereas in MNNG HOS cells, this effect is only hinted at.

Effects of Ly294002 and PD98059 on the Mas mRNA expression. The administration of Ly 294002 provoked a very consistent 2.4-fold increase of Mas mRNA amounts in both MNNG-HOS [2.212 (1.72; 2.89), $\mathrm{p}<0.05]$ and U-2 OS cells $[2.38(1.70 ; 4.11), \mathrm{p}<0.001]$. A similar increase could be observed in response to PD98059. Whereas a 2.1-fold increase
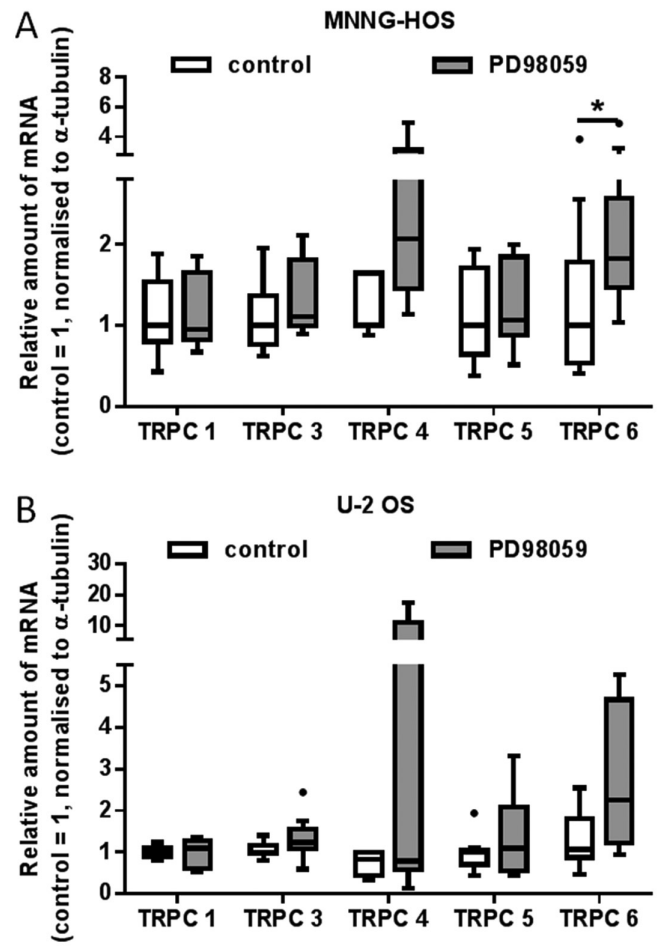

Figure 6. Effect of the MEK inhibitor PD98059 on the TRPC-mRNA content of (A) MNNG-HOS and (B) U-2 OS osteosarcoma cells. Cells were exposed to the MEK inhibitor PD98059 $(50 \mu \mathrm{M})$ for $24 \mathrm{~h}$. Subsequently, mRNA content was analyzed by reverse transcription-quantitative PCR. Data are presented as boxplots with medians, quartiles and an IQR $\pm 1.5 \times \mathrm{IQR}$ with outliers as indicated (Tukey's method; $\mathrm{n}=8 ;{ }^{*} \mathrm{P}<0.05$ ). IQR, interquartile range; TRPC, canonical transient receptor potential channels.

[2.1 (1.28; 3.33), $\mathrm{p}<0.05]$ in Mas mRNA amounts could be observed in U-2 OS cells, a 1.4-fold [1.38 $(0.93 ; 2.26), \mathrm{p}=0.08]$ increase in MNNG-HOS cells could be observed as a tendency only (Fig. 7).

Effects of AVE0991 or D-Ala ${ }^{7}$-Ang-(1-7) and TRPC inhibitors AC1903 or Pyr3 on the migration of MNNG-HOS and U-2 OS cells. The administration of Ang-(1-7) has been previously shown to reduce cell migration of U-2 OS cells without affecting that of MNNG-HOS cells in a Cultrex ${ }^{\circledR} 24$ Well Cell Migration Assay (16). In the present assay, this could be observed here as a tendency only: U-2 OS cells showed slightly less migration upon exposure to the Mas receptor agonist, AVE0991, and slightly increased migration when exposed to the antagonist, D-Ala ${ }^{7}$-Ang-(1-7) (A779; Fig. 8B). To assess the possible contribution of Mas-dependent TRPC activity modulation to alterations in cell migration, the inhibitors of TRPC5 or TRPC3, AC1903 or Pyr3, respectively, were applied alone or in combination with AVE0991 or A779.

In MNNG-HOS cells, the TRPC5 inhibitor, AC1903, on its own or in combination with A779 or AVE0991 did not affect cell migration (Fig. 8A). However, administration of the TRPC 3 inhibitor, Pyr3, significantly increased migration [Pyr3: 30.0 (28.6; 34.6) vs. DMSO: 25.8 (24.8; 27.48), p<0.05], an effect that could be further increased by simultaneous administration of AVE0991 [Pyr3+AVE0991: 33.7 (31.9; 35.6), $\mathrm{P}=0.087$ vs. Pyr3].

As shown in Fig. 8B, AC1903, although not affecting U-2 OS migration per se, caused a significant decrease of migra- 

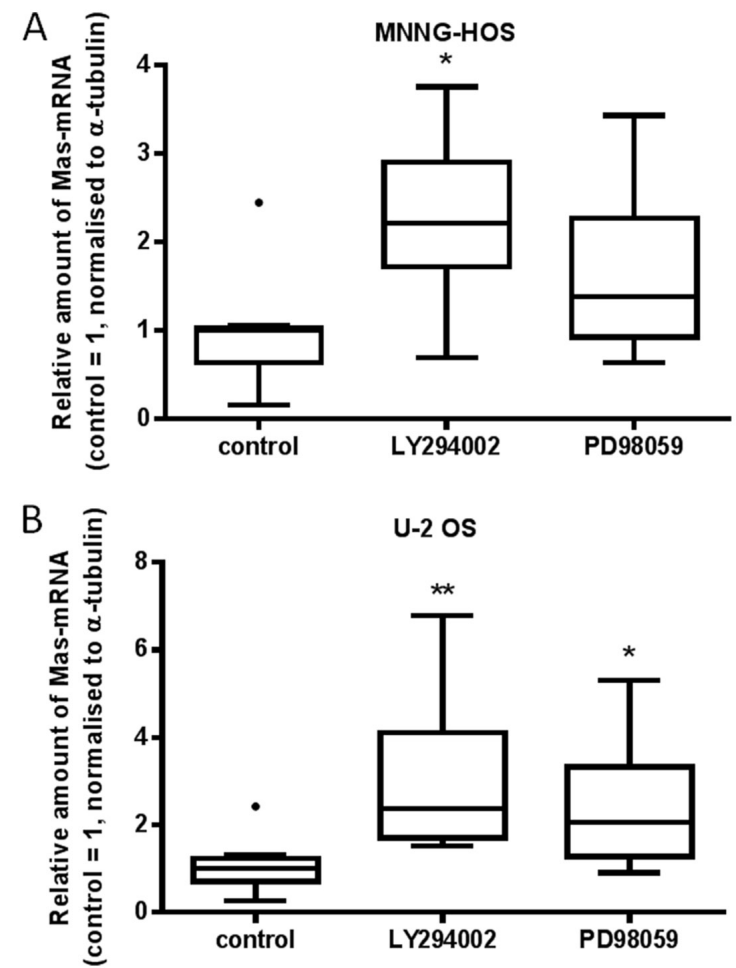

Figure 7. PD 98059 and LY294002 increase Mas mRNA content of (A) MNNG-HOS and (B) U-2 OS osteosarcoma cells. Cells were exposed to the MEK inhibitor, PD98059 $(50 \mu \mathrm{M})$, or PI3 kinase inhibitor, LY294002 $(100 \mu \mathrm{M})$, for $24 \mathrm{~h}$. Subsequently, mRNA content was determined by reverse transcription-quantitative PCR. Data are presented as boxplots with medians, quartiles and an IQR $\pm 1.5 \times \mathrm{IQR}$ with outliers as indicated (Tukey method; $\mathrm{n}=8 ;{ }^{*} \mathrm{P}<0.05,{ }^{* *} \mathrm{P}<0.01$ vs. control). IQR, interquartile range.

tion when applied together with A779. The administration of Pyr3 significantly reduced the migration of U-2 OS cells, an effect that could be prevented by the simultaneous administration of AVE0991.

\section{Discussion}

TRPCs transform various stimuli into appropriate cellular responses involving functions such as proliferation, migration, and survival. These processes are of paramount importance for normal growth, repair, and tissue homeostasis, and therefore their dysregulation is a feature typically observed during tumorigenesis and tumor progression. TRPCs have been associated with various diseases and, in particular, with cancer. With respect to the latter, altered expression, subcellular localization, and gene mutations of TRPC subtypes have been reported for an increasing number of tumor entities, including glioma as well as lung, breast, prostate, gastric, and hepatic cancer (reviewed in references 1,21-23). The abnormal regulation of the intracellular calcium balance certainly plays a major role here, but additional cellular processes, aside of enhancing $\mathrm{Ca}^{2+}$ entry, have been identified (22). Reinforcing this phenomenon, TRPC activity and/or expression could be modulated by inadequate signal input from aberrant GPCR or growth factor receptor activation in normal and (pre-)malignant cells. This has been observed for e.g. TRPC6, the membrane expression and activity of which appeared to be regulated by epidermal growth factor (EGF) receptor stimulation (24). Mechanistically,
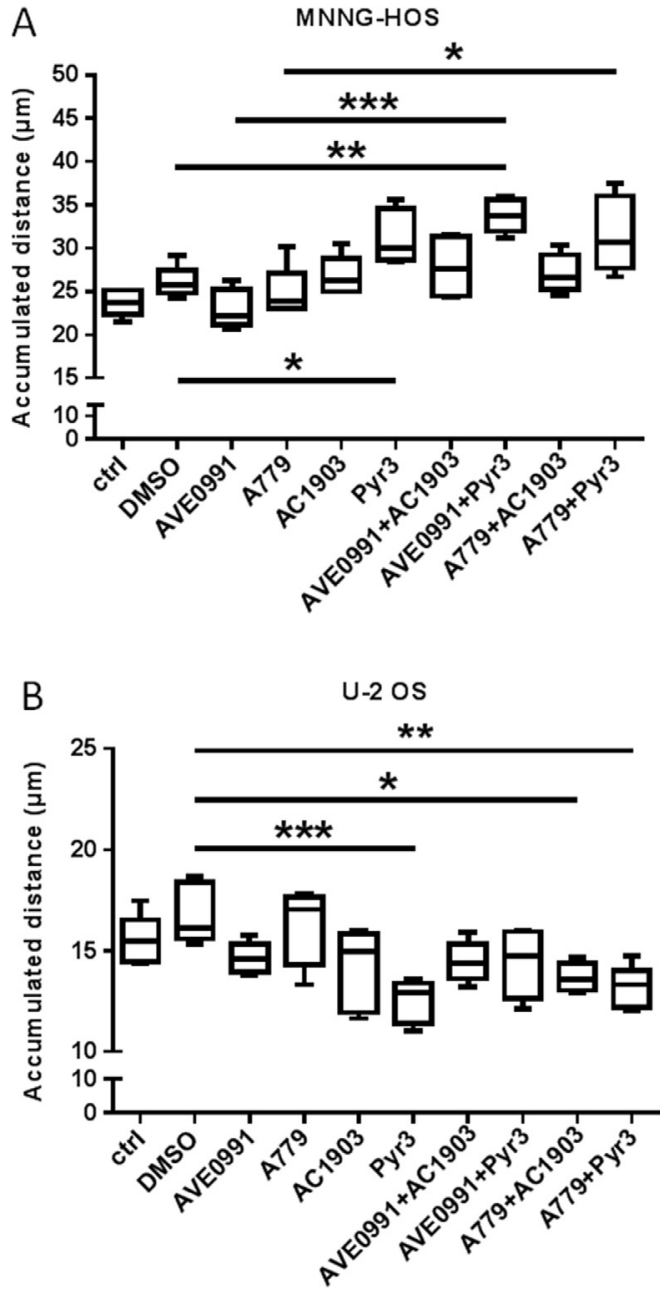

Figure 8. Effects of AVE0991, A779, AC1903 and Pyr3 on the migration of (A) MNNG-HOS and (B) U-2 OS osteosarcoma cells. Cells were exposed to AVE0991 (1 $\mu \mathrm{M})$, D-Ala ${ }^{\text {ets }}$-Ang-(1-7) $(1 \mu \mathrm{M})$, AC1903 (35 $\left.\mu \mathrm{M}\right)$, Pyr3 $(3.5 \mu \mathrm{M})$, or combinations thereof as indicated for $24 \mathrm{~h}$. Subsequently, migration was measured over $6 \mathrm{~h}$ (accumulated distance). Data are presented as boxplots with medians, quartiles and an IQR $\pm 1.5 \times$ IQR with outliers as indicated (Tukey method; $\mathrm{n}=5 ;{ }^{*} \mathrm{P}<0.05,{ }^{* *} \mathrm{P}<0.01,{ }^{* * * *} \mathrm{P}<0.001$ ). ctrl, control; $\mathrm{IQR}$, interquartile range.

a number of different signal transduction pathways mediate TRPC signaling including extracellular signal-regulated kinase Erk1/2, calcium/calmodulin-dependent protein kinase II $\alpha$ (CaMKII $\alpha)$, and calpain $(21,25)$. Furthermore, TRPCs 3, 4 and 5 have all been shown to contribute to cancer angiogenesis through modulating VEGF pathway $(26,27)$.

For cancer in general it is well established that dysregulation of $\mathrm{Ca}^{2+}$ signaling promotes tumor initiation, progression, metastasis, and angiogenesis (22). Little is known about the pathological relevance of dysregulated TRPC expression or activity specifically in osteosarcoma. It was previously shown that the knock-down of the GPCR, Mas, decreased the proliferation of MNNG-HOS and U-2 OS cells. In the present study, we examined the specific question of whether and to what extent this effect can be attributed to a Mas-dependent change in the TRPC expression. In addition, we investigated whether signaling pathways typically activated by GPCR or receptor tyrosine kinases are, in principle, able to influence TRPC expression in osteosarcoma cells. Selective inhibitors of the 
MEK/ERK1/2 and PI3 kinase signaling pathways, PD98059 and Ly294002, were used for this purpose.

The results of the present study show that neither the Mas receptor agonist, AVE0991 nor the antagonist, D-Ala ${ }^{7}$-Ang-(1-7), caused substantial changes in TRPC expression. The principle effectiveness of both compounds under the conditions applied was reflected in the marked change in the Mas-mRNA concentrations they provoked in both osteosarcoma cell lines. From this, it can be concluded that Mas-dependent regulation of osteosarcoma cell proliferation is not due to altered TRPC expression. However, the TRPC inhibitors applied here were capable of modulating effects of AVE0991 and A779 on osteosarcoma cell migration, which strongly implies that Mas-receptor signaling is associated with TRPC activity (rather than expression), and migration in osteosarcoma cell lines. Supporting this view are reports demonstrating: i) a cAMP-dependent increase in stimulated TRPC6 cation currents in TRPC6-expressing HEK293 cells, which could be diminished by LY294002 or PD 98059 (28); and ii) the increase of cAMP in response to Mas receptor agonists such as Ang-(1-7) observed in different cells $(18,29)$.

The increase in Mas mRNA levels in response to the antagonist very likely reflects a direct compensatory feedback mechanism, the equanimous effect of the non-peptide agonist can be explained as a similar response to functional desensitization following rapid activation of Mas (17).

The results of our study further show that inhibition of either PI3 kinase or MEK/Erk1/2 signal transduction provokes substantial changes in the mRNA expression of distinct TRPC subtypes. The administration of Ly294002 caused the strongest changes in the amounts of TRPC5-mRNA, which were induced 9.6-fold and 5.9-fold, respectively. In comparison, the induction of TRCP6 was less pronounced, whereas mRNA levels of TRPCs 1, 3 and 4 remained completely unaffected. The contribution of PI3 kinase signaling to TRPC6 expression has been described by others previously. However, in podocytes, vascular endothelial growth factor (VEGF) appeared to increase TRPC6 mRNA and protein levels, and this effect could be blocked by PI3 kinase inhibitors, including Ly294002 (30). As a more general mechanism, hormonal stimuli and growth factors via activation of TRPC activity and $\mathrm{Ca}^{2+}$ influx contribute to down-stream activation of MEK/ Erk1/2 or PI3 kinase signaling $(21,31)$. This has been shown for e.g. TRPC6 in hippocampal neurons and PC12 cells (32) and for TRPC3 in DT40 B-cells (33).

In the osteosarcoma cell lines MNNG-HOS and U-2 OS, the administration of the MEK/Erk inhibitor, PD98059, increased mRNA levels of TRPC6 whereas that of TRPC4 and TRPC 3 appeared to be affected only in one of the cell lines. Erk-dependent alterations in TRPC subtype expression have been observed by others in pulmonary arterial smooth muscle cells (34). Exposure of these cells to bone morphogenetic protein (BMP) 4 increased TRPC1, TRPC4 and TRPC6 expression, and this effect was abolished by pharmacological inhibition (PD98059) as well as by siRNA-mediated knockdown of Erk1/2 (34).

Our data consistently show a stimulatory effect of the pathway inhibitors on TRPC mRNA expression, which is inconsistent with the observations of other groups described before. It could be speculated, however, that different cell types react differently. In any case, this even applies to osteosarcoma cell lines from different species that differ in terms of their TRPC expression profile (14). Furthermore, each cell type has its special equipment with G-protein-coupled receptors (GPCRs), which can then result in very unique context- and stimulus-dependent activation patterns of TRPCs. It could be speculated that it is this difference in GPCRs that led to the different responses partially observed here for U-2 OS and MNNG HOS, a finding that reflects quite well the challenge posed with treating patients with 'individual' osteosarcoma. In our experiments, cells were exposed to the action of the inhibitors for a relatively long time, namely $24 \mathrm{~h}$. During this time, stimulatory feedback mechanisms may already be at play.

In accordance with a previous report (16), it could be demonstrated that the Mas receptor agonist AVE0991 negatively regulates U-2 OS cell migration, but is significantly increased in response to the Mas receptor antagonist A779.

In summary, the present study shows that Mas-dependent alterations in osteosarcoma cell line proliferation are not mediated by any changes in TRPC subtype gene expression. However, available data suggest that there are Mas-mediated changes in TRPC activity. TRPC activities have not been studied here, which is a clear limitation of our study. Another limitation is the use of only two cell lines, however, the previous observation that human osteosarcoma exhibit similar TRPC expression profiles (14) could be confirmed here. The results of our study show in principle and consistent with the literature, that: i) members of the TRPC family are abundantly expressed in osteosarcoma cell lines; and ii) that the cAMP-PI3K-PKBMEK-ERK $1 / 2$ signaling pathway examined can regulate the expression of TRPCs at the level of mRNA and may well contribute to Mas-dependent changes of TRPC activity and related functions including cell migration. TRPCs' potential relevance for diagnosis and, in particular, direct or signaling pathway-targeted treatment of osteosarcoma needs to be elucidated in further studies.

\section{Acknowledgements}

The authors would like to thank Mrs. Manja Möller and Mrs. Ines Schultz (Institute of Medical Biochemistry and Molecular Biology, University Medicine Greifswald, Greifswald, Germany) for their technical assistance.

\section{Funding}

No funding was received.

\section{Availability of data and materials}

The datasets used and/or analyzed during the current study are available from the corresponding author on reasonable request.

\section{Authors' contributions}

FL, SB, AK and CW performed the experiments. CW and UL were responsible for confirming the authenticity of all the raw data. FL, SB, AK, UL and CW conducted the data analyses. FL, CW and UL wrote the manuscript. UL and CW designed 
the study and planned experiments. All authors read and approved the final manuscript.

\section{Ethics approval and consent to participate}

Not applicable.

\section{Patient consent for publication}

Not applicable.

\section{Competing interests}

The authors declare that they have no competing interests.

\section{References}

1. Gautier M,Dhennin-Duthille I, Ay AS, Rybarczyk P, Korichneva I and Ouadid-Ahidouch $\mathrm{H}$ : New insights into pharmacological tools to TR(i)P cancer up. Br J Pharmacol 171: 2582-2592, 2014.

2. Wu LJ, Sweet TB and Clapham DE: International Union of Basic and Clinical Pharmacology. LXXVI. Current progress in the mammalian TRP ion channel family. Pharmacol Rev 62 : 381-404, 2010.

3. Capiod T: The need for calcium channels in cell proliferation. Recent Patents Anticancer Drug Discov 8: 4-17, 2013.

4. Hodeify R, Yu F, Courjaret R, Nader N, Dib M, Sun L, Adap E, Hubrack S and Machaca K: Regulation and role of store-operated $\mathrm{Ca}^{2+}$ entry in cellular proliferation. In: Calcium entry channels in non-excitable cells. Kozak JA and Putney JW Jr (eds). CRC Press/Taylor \& Francis, Boca Raton, FL, pp215-240, 2018.

5. Bomben VC and Sontheimer HW: Inhibition of transient receptor potential canonical channels impairs cytokinesis in human malignant gliomas. Cell Prolif 41: 98-121, 2008.

6. Cai R, Ding X, Zhou K, Shi Y, Ge R, Ren G, Jin Y and Wang Y: Blockade of TRPC6 channels induced G2/M phase arrest and suppressed growth in human gastric cancer cells. Int J Cancer 125: 2281-2287, 2009.

7. Hwang JA, Hwang MK, Jang Y, Lee EJ, Kim JE, Oh MH, Shin DJ, Lim S, Ji G, Oh U, et al: 20-O- $\beta$-d-glucopyranosyl20(S)-protopanaxadiol, a metabolite of ginseng, inhibits colon cancer growth by targeting TRPC channel-mediated calcium influx. J Nutr Biochem 24: 1096-1104, 2013.

8. Jiang HN, Zeng B, Zhang Y, Daskoulidou N, Fan H, Qu JM and Xu SZ: Involvement of TRPC channels in lung cancer cell differentiation and the correlation analysis in human non-small cell lung cancer. PLoS One 8: e67637, 2013.

9. Gaunt HJ, Vasudev NS and Beech DJ: Transient receptor potential canonical 4 and 5 proteins as targets in cancer therapeutics. Eur Biophys J 45: 611-620, 2016.

10. Huang Z, Fan G and Wang D: Downregulation of calbindin 1, a calcium-binding protein, reduces the proliferation of osteosarcoma cells. Oncol Lett 13: 3727-3733, 2017.

11. Wang Y, Yang Z, Meng Z, Cao H, Zhu G, Liu T and Wang X: Knockdown of TRPM8 suppresses cancer malignancy and enhances epirubicin-induced apoptosis in human osteosarcoma cells. Int J Biol Sci 10: 90-102, 2013.

12. Berna-Erro A, Redondo PC and Rosado JA: Store-operated $\mathrm{Ca}^{2+}$ entry. Adv Exp Med Biol 740: 349-382, 2012.

13. Clapham DE, Runnels LW and Strübing C: The TRP ion channel family. Nat Rev Neurosci 2: 387-396, 2001.

14. Huang YW, Chang SJ, Harn HI, Huang HT, Lin HH, Shen MR, Tang MJ and Chiu WT: Mechanosensitive store-operated calcium entry regulates the formation of cell polarity. J Cell Physiol 230: 2086-2097, 2015.

15. Abed E, Labelle D, Martineau C, Loghin A and Moreau R: Expression of transient receptor potential (TRP) channels in human and murine osteoblast-like cells. Mol Membr Biol 26: 146-158, 2009

16. Ender SA, Dallmer A, Lässig F, Lendeckel U and Wolke C: Expression and function of the ACE2/angiotensin(1-7)/Mas axis in osteosarcoma cell lines U-2 OS and MNNG-HOS. Mol Med Rep 10: 804-810, 2014.
17. Tirupula KC, Desnoyer R, Speth RC and Karnik SS: Atypical signaling and functional desensitization response of MAS receptor to peptide ligands. PLoS One 9: e103520, 2014.

18. Sahr A, Wolke C, Maczewsky J, Krippeit-Drews P, Tetzner A, Drews G, Venz S, Gürtler S, van den Brandt J, Berg S, et al: The angiotensin-(1-7)/Mas axis improves pancreatic $\beta$-cell function in vitro and in vivo. Endocrinology 157: 4677-4690, 2016.

19. Chilukoti RK, Mostertz J, Bukowska A, Aderkast C, Felix SB, Busch M, Völker U, Goette A, Wolke C, Homuth G, et al: Effects of irbesartan on gene expression revealed by transcriptome analysis of left atrial tissue in a porcine model of acute rapid pacing in vivo. Int J Cardiol 168: 2100-2108, 2013.

20. Livak KJ and SchmittgenTD: Analysis of relative gene expression data using real-time quantitative PCR and the $2(-\Delta \Delta \mathrm{C}(\mathrm{T}))$ Method. Methods 25: 402-408, 2001.

21. Chen J, Luan Y, Yu R, Zhang Z, Zhang J and Wang W: Transient receptor potential (TRP) channels, promising potential diagnostic and therapeutic tools for cancer. Biosci Trends 8: 1-10, 2014.

22. Bacsa B, Tiapko O, Stockner T and Groschner K: Mechanisms and significance of $\mathrm{Ca}^{2+}$ entry through TRPC channels. Curr Opin Physiol 17: 25-33, 2020.

23. Chen X, Sooch G, Demaree IS, White FA and Obukhov AG: Transient receptor potential canonical (TRPC) channels: Then and now. Cells 9: 2020

24. Odell AF, Scott JL and Van Helden DF: Epidermal growth factor induces tyrosine phosphorylation, membrane insertion, and activation of transient receptor potential channel 4. J Biol Chem 280: 37974-37987, 2005.

25. Xu J, Wang H, Hu Y, Zhang YS, Wen L, Yin F, Wang Z, Zhang Y, Li S, Miao Y, et al: Inhibition of CaMKII $\alpha$ activity enhances antitumor effect of fullerene C60 nanocrystals by suppression of autophagic degradation. Adv Sci (Weinh) 6: 1801233, 2019.

26. Asghar MY, Magnusson M, Kemppainen K, Sukumaran P, Löf C, Pulli I, Kalhori V and Törnquist K: Transient receptor potential canonical 1 (TRPC1) channels as Regulators of sphingolipid and VEGF receptor expression: Impolications for thyroid cancer cell migration and proliferation. J Biol Chem 290: 16116-16131, 2015.

27. Tao X, Zhao N, Jin H, Zhang Z, Liu Y, Wu J, Bast RC Jr, Yu Y and Feng Y: FSH enhances the proliferation of ovarian cancer cells by activating transient receptor potential channel C3. Endocr Relat Cancer 20: 415-429, 2013.

28. Shen B, Kwan HY, Ma X, Wong CO, Du J, Huang Y and Yao X: cAMP activates TRPC6 channels via the phosphatidylinositol 3-kinase (PI3K)-protein kinase B (PKB)-mitogen-activated protein kinase kinase (MEK)-ERK1/2 signaling pathway. J Biol Chem 286: 19439-19445, 2011.

29. Tetzner A, Gebolys K, Meinert C, Klein S, Uhlich A, Trebicka J, Villacañas Ó and Walther T: G-protein-coupled receptor MrgD is a receptor for angiotensin-(1-7) involving adenylyl cyclase, cAMP, and phosphokinase A. Hypertension 68: 185-194, 2016.

30. Thilo F, Liu Y, Loddenkemper C, Schuelein R, Schmidt A, Yan Z, Zhu Z,Zakrzewicz A, Gollasch M and Tepel M: VEGF regulates TRPC6 channels in podocytes. Nephrol Dial Transplant 27: 921-929, 2012.

31. Jardin I, Diez-Bello R, Lopez JJ, Redondo PC, Salido GM, Smani T and Rosado JA: TRPC6 channels are required for proliferation, migration and invasion of breast cancer cell lines by modulation of Orai1 and Orai3 surface exposure. Cancers (Basel) 10: 331, 2018.

32. Heiser JH, Schuwald AM, Sillani G, Ye L, Müller WE and Leuner K: TRPC6 channel-mediated neurite outgrowth in PC12 cells and hippocampal neurons involves activation of RAS/ MEK/ERK, PI3K, and CAMKIV signaling. J Neurochem 127: 303-313, 2013.

33. Numaga-Tomita T, Nishida M, Putney JW Jr and Mori Y: TRPC3 amplifies B-cell receptor-induced ERK signalling via protein kinase D-dependent Rap1 activation. Biochem J 473: 201-210, 2016.

34. Li X, Lu W, Fu X, Zhang Y, Yang K, Zhong N, Ran P and Wang J: BMP4 increases canonical transient receptor potential protein expression by activating $\mathrm{p} 38$ MAPK and ERK1/2 signaling pathways in pulmonary arterial smooth muscle cells. Am J Respir Cell Mol Biol 49: 212-220, 2013. 